\title{
Cornering compressed gluino at the LHC
}

\author{
Natsumi Nagata, ${ }^{a}$ Hidetoshi Otono $^{b}$ and Satoshi Shirai ${ }^{c}$ \\ ${ }^{a}$ Department of Physics, University of Tokyo, \\ Tokyo 113-0033, Japan \\ ${ }^{b}$ Research Center for Advanced Particle Physics, Kyushu University, \\ Fukuoka 819-0395, Japan \\ ${ }^{c}$ Kavli Institute for the Physics and Mathematics of the Universe (WPI), \\ The University of Tokyo Institutes for Advanced Study, The University of Tokyo, \\ Kashiwa 277-8583, Japan \\ E-mail: natsumi@hep-th.phys.s.u-tokyo.ac.jp, \\ otono@phys.kyushu-u.ac.jp, satoshi.shirai@ipmu.jp
}

ABSTRACT: We discuss collider search strategies of gluinos which are highly degenerate with the lightest neutralino in mass. This scenario is fairly difficult to probe with conventional search strategies at colliders, and thus may provide a hideaway of supersymmetry. Moreover, such a high degeneracy plays an important role in dark matter physics as the relic abundance of the lightest neutralino is significantly reduced via coannihilation. In this paper, we discuss ways of uncovering this scenario with the help of longevity of gluinos; if the mass difference between the lightest neutralino and gluino is $\lesssim 100 \mathrm{GeV}$ and squarks are heavier than gluino, then the decay length of the gluino tends to be of the order of the detector-size scale. Such gluinos can be explored in the searches of displaced vertices, disappearing tracks, and anomalously large energy deposit by (meta)stable massive charged particles. We find that these searches are complementary to each other, and by combining their results we may probe a wide range of the compressed gluino region in the LHC experiments.

Keywords: Supersymmetry Phenomenology

ARXIV EPRINT: 1701.07664 


\section{Contents}

1 Introduction 1

2 Properties of compressed gluino $\quad 3$

2.1 Gluino decay 3

$2.2 R$-hadrons 4

3 LHC searches $\quad 6$

3.1 ATLAS experiment 6

$\begin{array}{lll}3.2 & \text { ATLAS searches } & 7\end{array}$

3.3 LHC prospects 8

4 Conclusion and discussion $\quad 11$

\section{Introduction}

Supersymmetric (SUSY) extensions of the Standard Model (SM) have been thought of as the leading candidate of physics beyond the SM. In particular, weak-scale SUSY has various attractive features - the electroweak scale is stabilized against quantum corrections, gauge coupling unification is achieved, and so on - and therefore has widely been studied so far. This paradigm is, however, under strong pressure from the results obtained at the Large Hadron Collider (LHC). Direct searches of SUSY particles impose stringent limits on their masses, especially those of colored particles such as squarks and gluino [1-3]. In addition, the observed value of the mass of the SM-like Higgs boson [4-6], $m_{h} \simeq 125 \mathrm{GeV}$, may also imply that SUSY particles are rather heavy. In the minimal supersymmetric Standard Model (MSSM), the tree-level value of the SM-like Higgs boson mass is smaller than the $Z$-boson mass $[7,8]$, and we need sizable quantum corrections in order to explain the discrepancy between the tree-level prediction and the observed value. It turns out that sufficiently large radiative corrections are provided by stop-loop diagrams [9-13] if the stop masses are much larger than the electroweak scale.

The SUSY SM with heavy SUSY particles (or, equivalently, with a high SUSY-breaking scale) have various advantages from the phenomenological point of view [14-28]; $i$ ) severe limits from the measurements of flavor-changing processes and electric dipole moments can be evaded [29-33]; ii) heavy sfermions do not spoil successful gauge coupling unification if gauginos and Higgsinos remain around the $\mathrm{TeV}$ scale [34]; iii) the dimension-five proton decay caused by the color-triplet Higgs exchange [35, 36], which was problematic for weakscale SUSY [37, 38], is suppressed by sfermion masses and thus the current proton decay bound can be evaded if SUSY particles are heavy enough [39-47], making the minimal SUSY SU(5) grand unification [48, 49] viable; iv) cosmological problems in SUSY theories, 
such as the gravitino problem [50-54] and the Polonyi problem [55] can be avoided. Nevertheless, high-scale SUSY models may have a potential problem regarding dark matter. In SUSY SMs with $R$-parity conservation, the lightest SUSY particle (LSP) is stable and thus can be a dark matter candidate. In particular, if the LSP is the lightest neutralino, its relic abundance is determined by the ordinary thermal freeze-out scenario. Then, it turns out that if the mass of the neutralino LSP is well above the weak scale, its thermal relic abundance tends to exceed the observed value of dark matter density, $\Omega_{\mathrm{DM}} h^{2} \simeq 0.12$ [56]. Thus, the requirement of $\Omega_{\mathrm{DM}} h^{2} \lesssim 0.12$ imposes a severe constraint on models with high-scale SUSY breaking. ${ }^{1}$

In order to avoid over-production of the neutralino LSP in the high-scale SUSY scenario, it is necessary to assure a large annihilation cross section for the LSP. A simple way to do that is to assume the neutralino LSP to be an almost pure $\mathrm{SU}(2)_{L}$ multiplet, i.e., a wino or Higgsino. In such cases, the LSP has the electroweak interactions and thus has a relatively large annihilation cross section, which is further enhanced by the so-called Sommerfeld effects $[57,58]$. Indeed, the thermal relic abundance of wino and Higgsino with a mass of around $3 \mathrm{TeV}$ [59] and $1 \mathrm{TeV}$ [60], respectively, is found to be in good agreement with the observed dark matter density $\Omega_{\mathrm{DM}} h^{2} \simeq 0.12$ [56]. Smaller masses are also allowed by the observation; in such cases, their thermal relic accounts for only a part of the total dark matter density and the rest should be filled with other dark matter candidates and/or with non-thermal contribution via, e.g., the late-time decay of gravitinos [61, 62]. For previous studies of wino and Higgsino dark matter, see refs. [20, 57-59, 61-78] and refs. [79-87], respectively, and references therein.

On the other hand, bino-like dark matter in general suffers from over-production, and thus a certain mechanism is required to enhance the annihilation cross section. For example, we may utilize the $s$-channel resonant annihilation through the exchange of the Higgs bosons (called funnel) [88]. Coannihilation [88] may also work if there is a SUSY particle that is degenerate with the LSP in mass and has a large annihilation cross section; stau [89-92], stop [93-98], gluino [97-104], wino [63, 103, 105-107], etc., can be such a candidate. In particular, only the latter two can have a mass close to the LSP in the case of the split-SUSY type models [14-28]. In this paper, we especially focus on the neutralinogluino coannihilation case as this turns out to offer a variety of interesting signatures at colliders and thus can be probed in various search channels at the LHC. For a search strategy of the bino-wino coannihilation scenario at the LHC, see ref. [107].

In order for the neutralino-gluino coannihilation to work, the neutralino LSP and gluino should be highly degenerate in mass. For instance, if the neutralino LSP is bino-like, the mass difference between the LSP and gluino, $\Delta m$, needs to be less than around $100 \mathrm{GeV}$ and squark masses should be less than $\mathcal{O}(100) \mathrm{TeV}$ for coannihilation to be effective [104]. Such a small mass difference makes it difficult to probe this scenario in the conventional LHC searches as hadronic jets from the decay products of gluinos tend to be soft. In the previous work [104], however, it is pointed out that such a compressed gluino with heavy

\footnotetext{
${ }^{1}$ In fact, environmental selection with multiverse may naturally give the condition $\Omega_{\mathrm{DM}} h^{2} \lesssim 0.12$ and favor a "Spread SUSY"-type spectrum [19, 20, 28].
} 


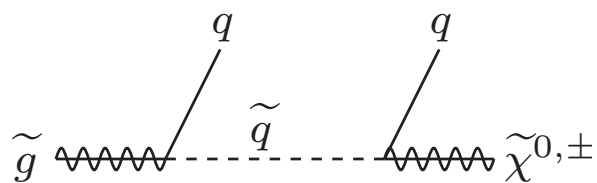

(a) Three-body decay.

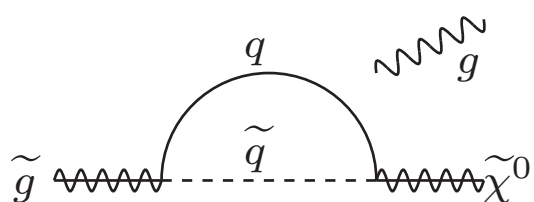

(b) Two-body decay.

Figure 1. Diagrams that give rise to the gluino decay.

squarks has a decay length of $\gtrsim \mathcal{O}(1) \mathrm{mm}$ and therefore may be probed by using searches for displaced vertices (DVs). In fact, it was shown in ref. [104] that the DV search at the LHC can investigate a wide range of parameter space where the correct dark matter abundance is obtained for the bino LSP through coannihilation with gluinos.

In this paper, we further study the prospects of the LHC searches to probe this compressed gluino scenario. In particular, we discuss search strategies for the very degenerate case, i.e., $\Delta m \lesssim \mathcal{O}(10) \mathrm{GeV}$. Such an extremely small mass difference considerably narrows down the reach of DV searches. We however find that for such a small mass difference, long-lived gluinos leave disappearing track signals when they form charged $R$-hadrons, and thus can be probed in the disappearing track searches. In addition, for gluinos which have a decay length of $\gtrsim 1 \mathrm{~m}$, searches for anomalously large energy deposit by (meta)stable heavy charged particles can be exploited. We see below that these three searches are complementary to each other. Hence, by combining the results from these searches we can thoroughly study the compressed gluino scenario at the LHC.

\section{Properties of compressed gluino}

Here we discuss the compressed gluino signatures at colliders. If the masses of squarks are very large and/or the mass difference between the gluino and the neutralino LSP $\Delta m$ is quite small, the gluino decay width is strongly suppressed and its decay length can be as large as the detector-size scale. We briefly discuss this feature in section 2.1. ${ }^{2}$ When the gluino lifetime is longer than the QCD hadronization time scale, gluinos produced at colliders form $R$-hadrons. We discuss the properties of $R$-hadrons and their implications on the LHC searches in section $2.2 .^{3}$

\subsection{Gluino decay}

The decay of gluinos is induced by the diagrams shown in figure 1. Here, we focus on the case where the mass difference $\Delta m$ is rather small $(\Delta m \lesssim 100 \mathrm{GeV})$ and squark masses are much larger than the electroweak scale; i.e., $\widetilde{m} \gtrsim 10 \mathrm{TeV}$, where $\widetilde{m}$ denotes the typical scale of squark masses. ${ }^{4}$ In this case, the tree-level three-body decay process shown in figure 1a is proportional to $(\Delta m)^{5}$, and thus its decay width is strongly suppressed for compressed

\footnotetext{
${ }^{2}$ For detailed discussions on the decay of (long-lived) gluinos, see refs. [108-115].

${ }^{3}$ For previous studies on the $R$-hadron properties in the split-SUSY, see refs. [116, 117].

${ }^{4}$ We however note that gluinos can be long-lived even though $\widetilde{m}$ is around the TeV scale if the mass difference $\Delta m$ is small enough. This possibility is briefly discussed in section 4 .
} 
gluinos. For the loop-induced two-body decay in figure 1b, on the other hand, the decay width strongly depends not only on the mass difference but also on the nature of the lightest neutralino. If the neutralino LSP is a pure bino and left-right mixing of the scalar top quarks is small, the matrix element of the two-body decay process is suppressed by a factor of $m_{\widetilde{g}}-m_{\widetilde{B}}\left(m_{\widetilde{g}}\right.$ and $m_{\widetilde{B}}$ are the gluino and bino masses, respectively), which originates from a chirality flipping in the external lines. As a result, the two-body decay rate also goes as $\Gamma(\widetilde{g} \rightarrow \widetilde{B} g) \propto(\Delta m)^{5}$, which makes the three-body decay channel dominate the two-body one. If the LSP is a pure wino, the two-body decay is suppressed by the $\mathrm{SU}(2)_{L}$ gauge symmetry, and thus the three-body decay is again the dominant channel. Contrary to these cases, if the LSP is Higgsino-like, the two-body decay is the main decay process. In this case, the dominant contribution to this decay process comes from the top-stop loop diagram and its matrix element is proportional to the top mass $m_{t}$. Thus, the two-body decay rate goes as $\Gamma\left(\widetilde{g} \rightarrow \widetilde{H}^{0} g\right) \propto m_{t}^{2}(\Delta m)^{3}$. In addition, this loop contribution is logarithmically enhanced as the masses of the scalar top quarks get larger. The tree-level decay process is, on the other hand, suppressed by small Yukawa couplings since the stop exchange process, which is large because of the top Yukawa coupling, is kinematically forbidden for compressed gluinos.

Eventually, we see that the gluino decay length is approximately given by

$$
c \tau_{\widetilde{g}}=\left\{\begin{array}{ll}
\mathcal{O}(1-10) \mathrm{cm} \times\left(\frac{\Delta m}{10 \mathrm{GeV}}\right)^{-5}\left(\frac{\widetilde{m}}{10 \mathrm{TeV}}\right)^{4} \quad(\text { bino or wino LSP }) \\
\mathcal{O}(0.01-0.1) \mathrm{mm} \times\left(\frac{\Delta m}{10 \mathrm{GeV}}\right)^{-3}\left(\frac{\widetilde{m}}{10 \mathrm{TeV}}\right)^{4} \quad(\text { Higgsino LSP })
\end{array} .\right.
$$

The dependence of these approximated formulae on $\Delta m$ is captured by the above discussions. From these expressions, we find that compressed gluinos generically have decay lengths of the order of the detector size when the squark mass scale is $\gtrsim 10 \mathrm{TeV}$. We make the most of this observation to probe the compressed gluino scenario.

There are several situations where the above tendency of gluino decay may be altered. For the bino LSP case, the two-body decay channel can be important if the left-right mixing in the scalar top sector is large and $\Delta m$ is very small. This decay branch can also be enhanced if there is a sizable bino-Higgsino mixing. In addition, if $\Delta m \ll 10 \mathrm{GeV}$, the parton-level description gets less appropriate, and the hadronic properties of decay products significantly affect the gluino decay. Further dedicated studies are required to give a precise theoretical calculation of the gluino decay rate for this very small $\Delta m$ region, which is beyond the scope of this paper.

\section{$2.2 \quad R$-hadrons}

A long-lived gluino forms a bound state with quarks and/or gluons once they are produced at colliders. Such bound states, being $R$-parity odd, are called $R$-hadrons [118]. $R$-hadrons are categorized into several classes in terms of their constituents; if $R$-hadrons are composed of a gluino and a pair of quark and anti-quark, $\widetilde{g} \bar{q} q$, they are called $R$-mesons; if they consist of a gluino and three quarks (anti-quarks), $\widetilde{g} q q q(\widetilde{g} \bar{q} \bar{q} \bar{q})$, they are called $R$-baryons 
( $R$-antibaryons); a bound state which is made of a gluino and a gluon, $\widetilde{g} g$, is referred to as an $R$-glueball.

The production fractions of $R$-hadron species have a direct impact on the gluino search sensitivities discussed below, as some of these search strategies rely on the production of charged $R$-hadrons. A computation [119] in which hadronization is performed using Pyтhia [120] shows that the production rates of $R$-mesons dominate those of $R$-baryons. The production fraction of $R$-glueball is, on the other hand, theoretically unknown and thus regarded as a free parameter. In the analysis of ref. [119], this fraction is set to be $10 \%$, which is the default value used in PyтнIA. Then, it is found that the fraction of $R$-mesons is $88.5 \%$ while that of $R$-baryons is only $1.6 \%$. Among them, charged $R$-hadrons are $44.8 \%$. This value however significantly decreases if we set the $R$-glueball fraction to be a larger value. Taking this ambiguity into account, in the following analysis, we take different values for the $R$-glueball fraction and regard the resultant changes as theoretical uncertainty.

The mass spectrum of $R$-hadrons, especially that of $R$-mesons and $R$-glueball, also affects the $R$-hadron search strategy significantly. An estimation [121] based on a simple mass formula for the lowest hadronic states [122], which is derived from the color-spin interaction given by one gluon exchange, indicates that the lightest $R$-meson state is " $R$ rho", namely, a bound state which consists of a gluino and a vector iso-triplet made of up and down quarks. The mass of " $R$-pion" (a bound state of a gluino and a spin-zero iso-triplet made of up and down quarks) is found to be larger than the $R$-rho mass by about $80 \mathrm{MeV}$. This observation is consistent with the calculations using the bag model [123] and QCD lattice simulation [124], which predict $R$-rho to be lighter than $R$-pion by $40 \mathrm{MeV}$ and $50 \mathrm{MeV}$, respectively. On the other hand, there is controversy about the estimation of the $R$-glueball mass. An estimation by means of the constituent masses of partons shows that $R$-glueball is heavier than $R$-rho by $120 \mathrm{MeV}$ [119]. The bag-model calculation [123] also predicts $R$-glueball to be slightly heavier than $R$-rho. However, the lattice result [124] shows that the $R$-rho mass is larger than the $R$-glueball mass by $47 \mathrm{MeV}$, though we cannot conclude by this result that these results are incompatible with each other since the uncertainty of this calculation is as large as $90 \mathrm{MeV}$ (and the former two estimations also suffer from uncertainties of similar size). We here note that if $R$-glueball is lighter than $R$-rho and the mass difference between them is larger than the pion mass, then an $R$-rho can decay into an $R$-glueball and a pion via strong interactions. Other $R$-mesons may also decay into $R$-glueball. This considerably reduces the number of tracks associated with charged $R$-mesons, and thus weakens the discovery reach of $R$-hadrons. In the following analysis, we assume that such decay channels are kinematically forbidden, as is supported by the above calculations of the $R$-meson and $R$-glueball mass spectrum. As for $R$-baryons, the flavor singlet $J=0$ state, which has a non-zero strangeness, is the lightest $[125,126]$. In addition, there are flavor octet states which are stable against strong decays and heavier than the singlet state by about a few hundred MeV. Their weak-decay lifetime is likely to be sufficiently long so that they can be regarded as stable at colliders [127].

While $R$-hadrons are propagating through a detector, they may scatter off nuclei in it. Such processes are potentially important since they may change $R$-hadron species. For instance, by scattering a nucleon in the detector material, an $R$-meson or $R$-glueball 
can be converted into an $R$-baryon with emitting a pion. However, the reverse process is unlikely since pions are rarely found in the detector material and the process itself suffers from kinematical suppression. For this reason, although $R$-mesons and $R$-glueball are dominantly produced at the outset, we have a sizable fraction for $R$-baryons in the outer part of detectors, such as in the Muon Spectrometer. The nuclear reaction rates of $R$-hadrons are evaluated in refs. [121, 127-129], and it is found that an $R$-hadron interacts with nucleons about five times while propagating in $2 \mathrm{~m}$ of iron. Therefore, most of $R$ mesons and $R$-glueballs may be converted into $R$-baryons before they enter into the Muon Spectrometer. In the analysis discussed below, however, we focus on the $R$-hadron searches using the Inner Detector, on which the nucleon interactions have little impact as the matter density up to the Inner Detector region is very low. This makes our search strategies free from uncertainties originating from the estimation of $R$-hadron interactions in detectors we neglect these effects in the following analysis.

\section{LHC searches}

Next, we discuss the LHC signatures of the compressed gluinos. Depending on the gluino lifetime and the gluino-LSP mass difference, we need adopt several strategies to catch gluino signals. In this paper, we focus on the ATLAS detector. The performance of the CMS detector is similar to that of the ATLAS, though.

\subsection{ATLAS experiment}

The ATLAS detector is located at one of the interaction points of the LHC, which consists of the Inner Detector, the calorimeters, the Muon Spectrometer, and the magnet systems [130]. The long-lived gluino searches discussed in this paper make full use of the Pixel detector and the SemiConductor Tracker (SCT) in the Inner Detector. Various dedicated techniques, which have been developed to search for new long-lived particles, can be applied to the long-lived gluino searches in order to maximize its discovery potential. Here, we briefly review the detectors used in the searches relevant to this work.

Pixel detector. The Pixel detector is the sub-detector closest to the interaction point, which has a four-layer cylindrical structure with a length of about $800 \mathrm{~mm}$ in the barrel region. The innermost layer called Insertable B-Layer (IBL), which was installed before the LHC-Run2 started [131], has silicon pixel sensors of $50 \times 250 \mu \mathrm{m}$ and is located at a radius of $33 \mathrm{~mm}$. The other layers have silicon pixel sensors of $50 \times 400 \mu \mathrm{m}$ at radii of $50.5 \mathrm{~mm}, 88.5 \mathrm{~mm}$, and $122.5 \mathrm{~mm}$. The Pixel detector can measure the energy deposit along the trajectory of each charged particle, i.e., $d E / d x$, which is sensitive to slow-moving (meta)stable particles according to the Bethe-Bloch formula.

SCT. The SCT surrounds the Pixel detector, which has four layers in the barrel region with a length of about $1500 \mathrm{~mm}$ at radii of $299 \mathrm{~mm}, 371 \mathrm{~mm}, 443 \mathrm{~mm}$, and $514 \mathrm{~mm}$. A module on each layer consists of two $80 \mu \mathrm{m}$-pitch strip silicon sensors with a stereo angle of $40 \mathrm{mrad}$ between strip directions. The SCT does not have a capability to measure $d E / d x$ in contrast to the Pixel detector, since the SCT employs binary readout architecture. 


\subsection{ATLAS searches}

Gluinos give rise to various signatures at colliders depending on the decay lifetime. In this work, we consider the following four search strategies ${ }^{5}$ which are sensitive to different ranges of the gluino decay length:

Prompt decay [1]: $\boldsymbol{c} \boldsymbol{\tau}_{\widetilde{g}} \lesssim \mathbf{1} \mathbf{m m}$. Searches for a new particle which is assumed to decay at the interaction point are also sensitive to long-lived particles. The ATLAS experiment reconstructs tracks whose transverse impact parameters $\left(d_{0}\right)$ are less than $10 \mathrm{~mm}$, then checks a correspondence with primary vertices. As a result, a portion of the decay vertices of long-lived particles is merged with one of the primary vertices.

Generally, for metastable gluinos, these inclusive searches get less effective for $c \tau_{\widetilde{g}} \gg$ $1 \mathrm{~cm}$, since jets from the gluino decay are displaced from the primary vertex and fail the event selection criteria [136]. In the compressed gluino case, however, these searches are less sensitive to the gluino lifetime, since it is jets from the initial state radiation that play a main role in the conventional jets + MET searches. For this reason, even for a gluino with a decay length greater than $\mathcal{O}(1) \mathrm{m}$, the resultant mass bound will be similar to that for a prompt decay compressed gluino.

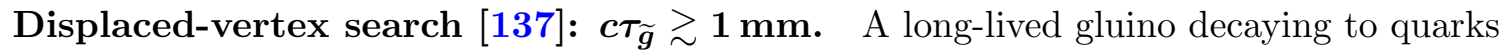
or a gluon leaves a displaced vertex (DV) away from the interaction point. In order to reconstruct tracks from such a DV, the requirement on the transverse impact parameter for tracks is loosen such that $2 \mathrm{~mm}<\left|d_{0}\right|<300 \mathrm{~mm}$. As a result, the sensitivity is maximized for particles with a decay length of $\mathcal{O}(10) \mathrm{mm}$.

The sensitivity becomes worse as the mass difference between gluino and the LSP gets smaller [104], since the invariant mass of DVs is required to be larger than $10 \mathrm{GeV}$ in order to separate signal events from background fake vertices. Due to this requirement, the gluino-LSP mass difference of $\lesssim 20 \mathrm{GeV}$ is hard to probe.

Disappearing-track search [138]: $c \tau_{\widetilde{g}} \gtrsim \mathbf{1 0} \mathrm{cm}$. Originally, the disappearing-track search has been developed for the search of long-lived charged winos with the neutral wino being the LSP [21, 139-142]. This technique may also be applicable to long-lived gluinos for the following reason. As we discuss in the previous section, a certain fraction of long-lived gluinos form charged $R$-hadrons. If the gluino and the LSP are degenerate in mass, the track associated with a charged $R$-hadron seems to disappear when the gluino in the $R$-hadron decays, since the jet emission from the gluino decay is very soft. As mentioned above, the DV search does not work efficiently when the gluino-LSP mass difference is very small. Such a degenerate mass region can instead be covered by the disappearing-track search.

A candidate track in the disappearing-track search should have four hits in the Pixel detector and the SCT with no activity after the last hit required. Thanks to the installation of IBL, the minimum length of disappearing tracks which can be searched for by this strategy is shorten from $299 \mathrm{~mm}$ to $122.5 \mathrm{~mm}$ in the LHC Run2. This allows the range of

\footnotetext{
${ }^{5}$ Some of these search strategies have already been considered in the context of long-lived gluino searches in refs. [104, 132]. For recent LHC studies on the compressed gluino scenario, see refs. [133-135].
} 


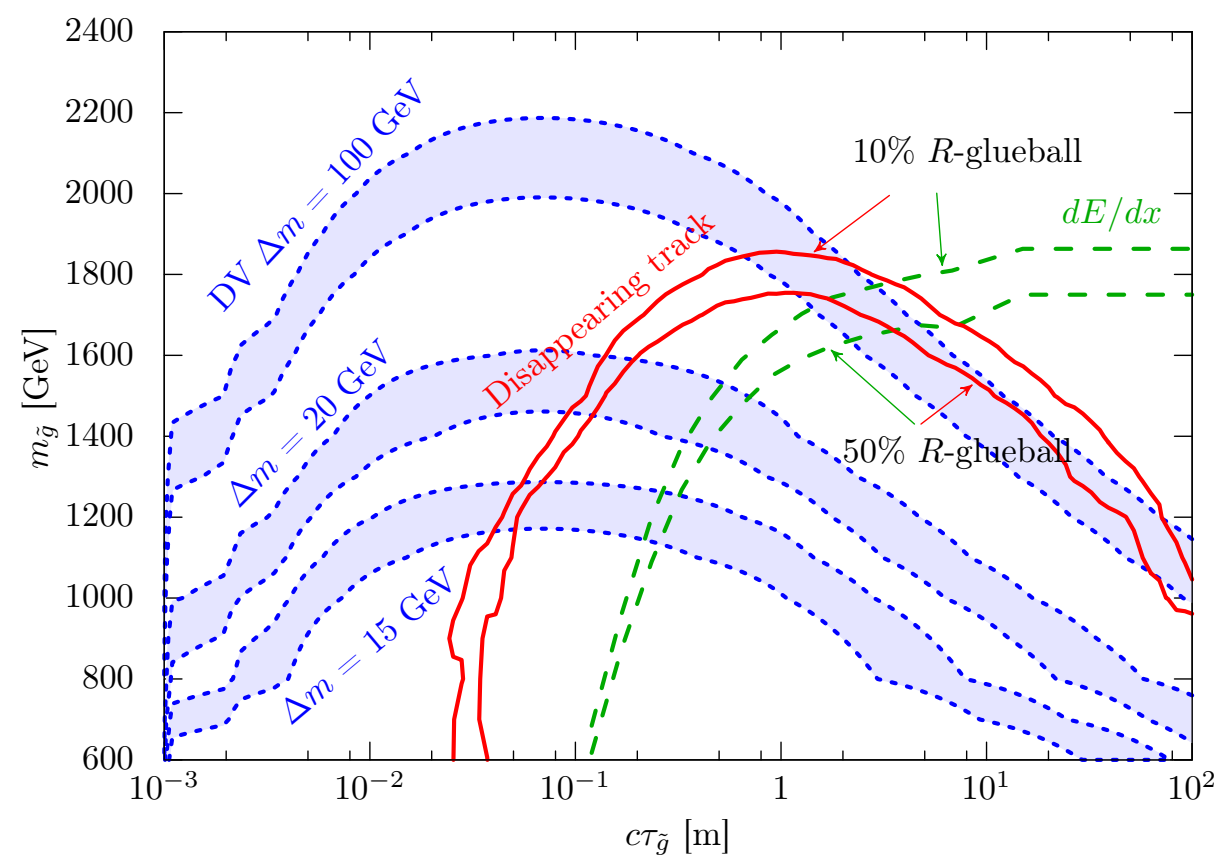

Figure 2. The expected limits from the searches considered in section 3.2 with an integrated luminosity of $40 \mathrm{fb}^{-1}$ at the $13 \mathrm{TeV}$ LHC. The blue dashed, red solid, and green long-dashed lines correspond to the DV search, the disappearing-track search, and the Pixel $d E / d x$ search, respectively.

decay lengths covered by the disappearing-track search to be slightly wider than that in the DV search.

Pixel $\boldsymbol{d E} / \boldsymbol{d} \boldsymbol{x}$ search [143]: $\boldsymbol{c} \tau_{\widetilde{g}} \gtrsim \mathbf{1} \mathrm{m}$. A particle with a mass of the order of the electroweak scale or larger tends to travel with a low velocity after it is produced at the LHC, which may be observed as a large $d E / d x$ in the Pixel detector. Hence, by searching for this signature, we can probe charged $R$-hadrons. While a minimum ionization particle is expected to give $\sim 1.2 \mathrm{MeV} \cdot \mathrm{cm}^{2} / \mathrm{g}$ of $d E / d x$, the threshold for the Pixel $d E / d x$ search is set to be $1.8 \mathrm{MeV} \cdot \mathrm{cm}^{2} / \mathrm{g}$ with a small correction depending on $\eta$. For the track selection, at least seven hits in the Pixel detector and the SCT are required, which corresponds to the minimum track-length of $371 \mathrm{~mm}$. Note that this search strategy does not require decay of particles, and thus is also sensitive to completely stable particles. For this reason, the cover-range of decay lengths by the Pixel $d E / d x$ search is quite broad.

\subsection{LHC prospects}

Now, we show the sensitivities of the searches listed in section 3.2 to the compressed gluino scenario. In this study, we use the program MADGRAPH5 [144]+Pythia8 [120, 145]+DELPHES3 [146] and estimate the production cross sections of SUSY particles with Prospino2 [147] or NLL-FAst [148-152].

In figure 2 , we show the prospects of each search in the $c \tau_{\tilde{g}}-m_{\tilde{g}}$ plane with an integrated luminosity of $40 \mathrm{fb}^{-1}$ at the $13 \mathrm{TeV}$ LHC. The blue dashed lines show the expected limits from the DV search with the gluino-LSP mass difference set to be $\Delta m=100 \mathrm{GeV}, 20 \mathrm{GeV}$, 
and $15 \mathrm{GeV}$ from top to bottom. We use the same event selection requirements as in the $8 \mathrm{TeV}$ study [107] except that we require missing energy $E_{\mathrm{T}}^{\text {miss }}$ to be greater than $200 \mathrm{GeV}$ as a trigger, which was $E_{\mathrm{T}}^{\text {miss }}>100 \mathrm{GeV}$ in the $8 \mathrm{TeV}$ study [107]. We expect the number of background events for this signal is as small as in the case of the $8 \mathrm{TeV}$ run [137]; here, we assume it to be $0-10$ and the systematic uncertainty of the background estimation to be $10 \%$. The upper (lower) border of each band corresponds to the case where the number of the background events is 0 (10). It is found that the sensitivity of the DV search is maximized for a gluino with a decay length of $\sim 10 \mathrm{~cm}$, and may reach a gluino mass of about $2.2 \mathrm{TeV}(1.2 \mathrm{TeV})$ for $\Delta m=100 \mathrm{GeV}(15 \mathrm{GeV})$. We also find that the DV search becomes less powerful when the gluino-LSP mass difference gets smaller, as mentioned in section 3.2.

The red solid lines show the expected reaches of the disappearing-track search. As we discussed above, this search relies on the production of charged $R$-hadrons, but the estimate of the charged $R$-hadron production rates suffers from uncertainty due to the unknown $R$-glueball fraction. To take this uncertainty into account, in figure 2 , we set the $R$-glueball fraction to be $10 \%$ and $50 \%$ in the top and bottom lines, respectively.

To estimate this prospect, we refer to the preliminary analysis of ref. [153] for the charged wino search at the LHC Run2, which is upgraded from the Run1 analysis [154]. These two adopt the same event topology - missing energy from initial state radiation and a disappearing charged track - and the analyses are essentially the same. We thus basically perform the same analysis as in the charged wino search at the LHC Run1 [154]. However, due to the improvement of the tracking system and increment of the beam energy, there are several changes in the Run2 analysis. First, as discussed in the previous subsection, the IBL enables a disappearing track to be detected with the Pixel detector only, which significantly increases the acceptance of shorter charged tracks. In the present analysis, we assume the detection efficiency $60 \%$ for $|\eta|<1.5$ and $13<r<30 \mathrm{~cm}$, which was zero in the Run1 study [154], and set the detection efficiency in the other regions to be the same as in the Run1 analysis. Another important difference is that if tracks are reconstructed with only the Pixel detector then the momentum resolution of these charged tracks is much worse than that in the Run1 analysis - since the travel length of such a charged track is very short, its momentum resolution gets worse: $\delta\left(1 / P_{\mathrm{T}}\right) \sim 1 / 8 \mathrm{TeV}^{-1}$. In order to avoid background contamination in signal events, the selection criterion for the transverse momentum of a charged track is fixed to be $100 \mathrm{GeV}$. Because of the change in the event trigger, the kinematic requirements are also changed such that $E_{\mathrm{T}}^{\mathrm{miss}}>140 \mathrm{GeV}$ and the leading jet with a transverse momentum of $P_{\mathrm{T}}>140 \mathrm{GeV}$. These three modifications in the event selection cuts and the additional acceptance region for disappearing tracks are the major changes from the Run1 analysis. According to ref. [153], after the above cuts are applied, the background mainly comes from $W+$ jets events in which a lepton or hadron is misidentified as disappearing tracks. The expected number of background events is $5.3 \pm 0.7$ for an integrated luminosity of $18.9 \mathrm{fb}^{-1}$. In the present analysis, the number of background events is assumed to be 10 and its uncertainty is supposed to be $10 \%$ for $40 \mathrm{fb}^{-1}$.

The result in figure 2 shows that the disappearing track search is sensitive to a decay length of $\sim 1 \mathrm{~m}$, and can probe a longer lifetime region than the DV search. Notice that even for $c \tau_{\tilde{g}}=\mathcal{O}(10) \mathrm{cm}$, around which the sensitivity of the DV search is maximized, the 


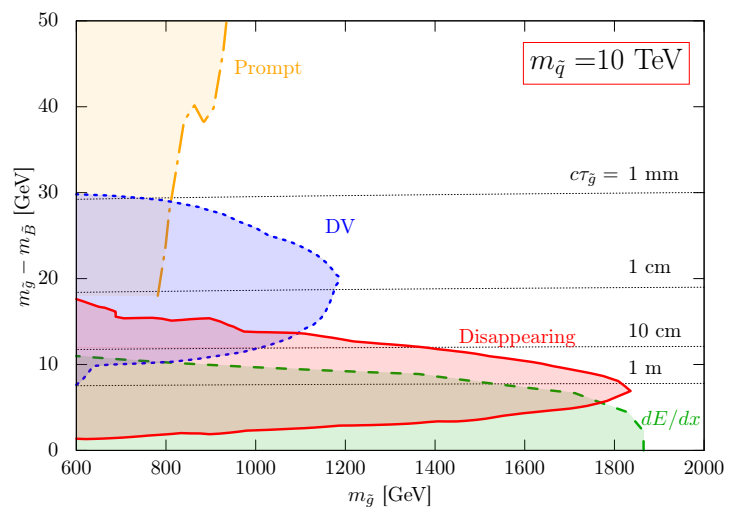

(a) $m_{\tilde{q}}=10 \mathrm{TeV}$.

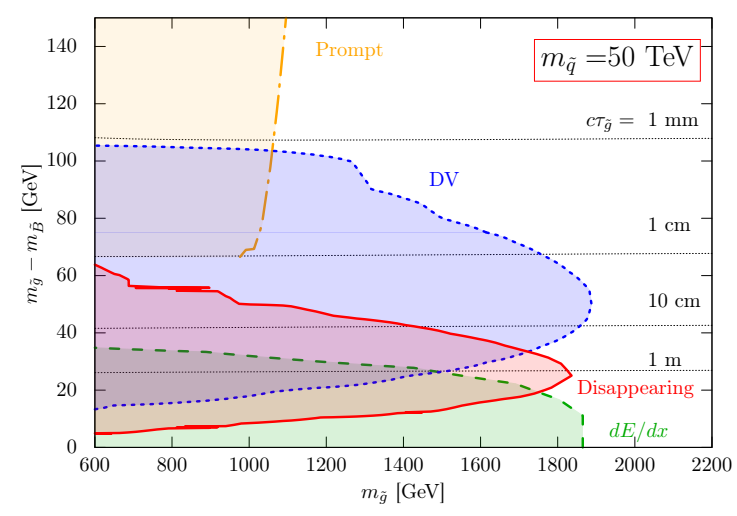

(b) $m_{\tilde{q}}=50 \mathrm{TeV}$.

Figure 3. The expected limits on the compressed gluino parameter space from the $13 \mathrm{TeV}$ LHC with an integrated luminosity of $40 \mathrm{fb}^{-1}$. The shaded areas with the blue dashed, red solid, green long-dashed, and orange dash-dotted borders can be probed with the searches of DVs, disappearing tracks, anomalous energy deposit $(d E / d x)$ in the Pixel detector, and prompt gluino decays, respectively. The black dotted lines show a contour plot for the gluino decay length $c \tau_{\tilde{g}}$.

reach of the disappearing-track search may exceed that of the DV search if the gluino-LSP mass difference is very small.

The expected reach of the Pixel $d E / d x$ search is plotted in the green long-dashed lines. Here again, we set the $R$-glueball fraction to be $10 \%$ and $50 \%$ in the top and bottom lines, respectively, to show the uncertainty from the unknown $R$-glueball fraction. For this analysis, we adopt the same event selection as in the study of ATLAS with $\sqrt{s}=13 \mathrm{TeV}$ and an integrated luminosity of $3.2 \mathrm{fb}^{-1}$ [155], where the $R$-glueball fraction is set to be $10 \%$. We have estimated the number of background events by rescaling the result of $3.2 \mathrm{fb}^{-1}$ to $40 \mathrm{fb}^{-1}$. We have also assumed that the gluino-LSP mass difference is so tiny that the missing energy, which is required in ref. [155], comes from initial state radiations. As can be seen, the Pixel $d E / d x$ search can probe $c \tau_{\tilde{g}} \gtrsim 1 \mathrm{~m}$, and its sensitivity does not decrease for $c \tau_{\tilde{g}} \gg 1 \mathrm{~m}$, contrary to the previous two cases.

To show the prospects of the above searches for probing the compressed gluino scenario, in figure 3, we show their expected reaches with the $13 \mathrm{TeV} 40 \mathrm{fb}^{-1} \mathrm{LHC}$ data in the $m_{\tilde{g}^{-}} \Delta m$ plane, where the shaded areas with the blue dashed, red solid, green long-dashed, and orange dash-dotted borders correspond to the searches of DVs, disappearing tracks, anomalous energy deposit $(d E / d x)$ in the Pixel detector, and prompt gluino decays, respectively. We also show a contour plot for the gluino decay length $c \tau_{\tilde{g}}$ in the black dotted lines. All squark masses (collectively denoted by $m_{\tilde{q}}$ ) are set to be $10 \mathrm{TeV}$ and $50 \mathrm{TeV}$ in figures $3 \mathrm{a}$ and $3 \mathrm{~b}$, respectively, and the LSP is assumed to be a pure bino. To obtain the expected sensitivity of the prompt gluino decay search with the $40 \mathrm{fb}^{-1}$ data, we adopt the event cut criteria used in ref. [1] and estimate the number of background events by rescaling the result of the $13.3 \mathrm{fb}^{-1}$ case. These figures show that the highly compressed region can be probed with the disappearing-track search and the Pixel $d E / d x$ search, while if the gluino-LSP mass splitting is large enough ( 


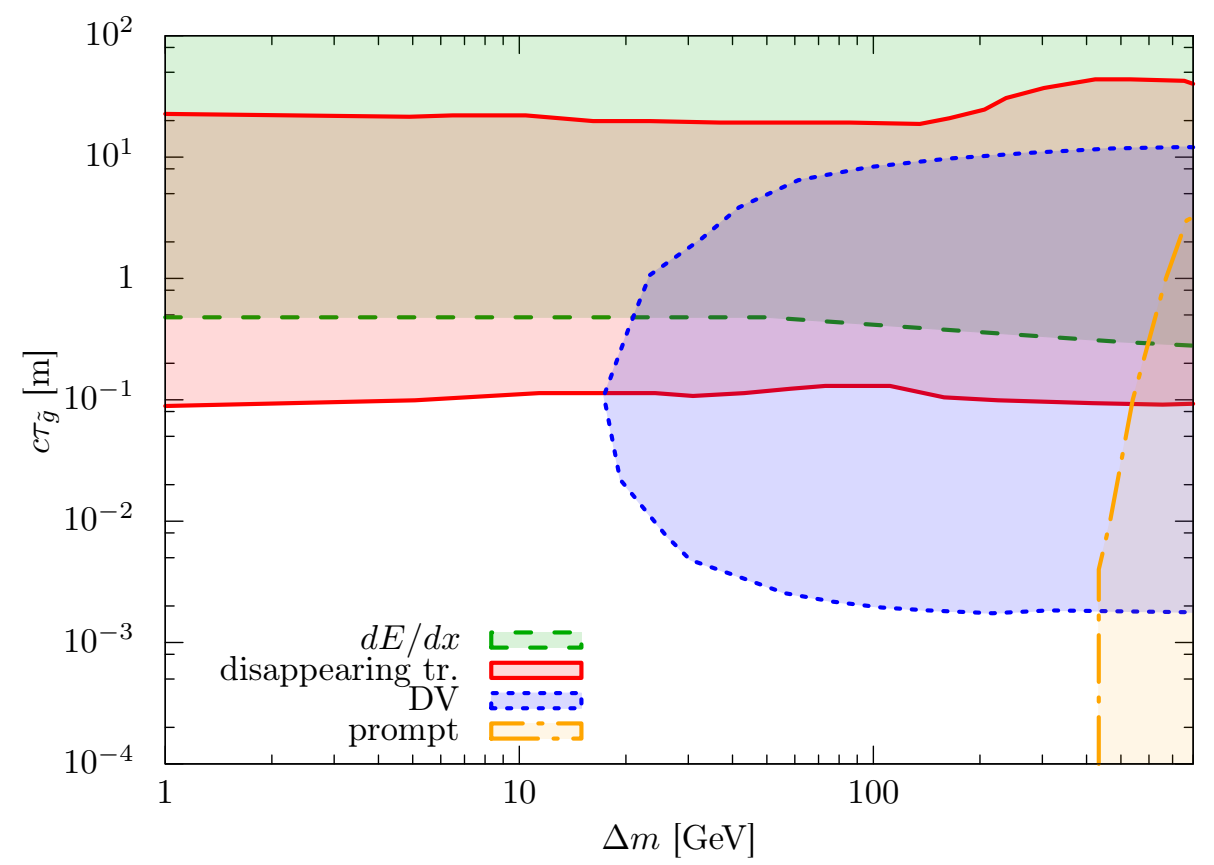

Figure 4. Testable regions of the gluino-LSP mass difference $\Delta m$ and the gluino decay length $c \tau_{\widetilde{g}}$ from the $d E / d x$, disappearing track, displaced vertex, and prompt inclusive searches at $13 \mathrm{TeV}$ LHC with an integrated luminosity of $40 \mathrm{fb}^{-1}$. Here we fix the gluino mass to be $1.5 \mathrm{TeV}$.

gluino searches can have sensitivities. Between these two regions, the DV search offers the best sensitivity. We also find that the reach of the DV search strongly depends on the squark masses. To obtain a gluino decay length of $c \tau_{\tilde{g}} \sim 10 \mathrm{~cm}$, to which the DV search is most sensitive, a smaller $\Delta m$ is required for lighter squarks (see eq. (2.1)). As noted above, the sensitivity of the DV search is considerably reduced for a small $\Delta m$; for this reason, the reach of the DV search shrinks for small squark masses. On the other hand, the disappearing-track and Pixel $d E / d x$ searches are rather robust on the change of $m_{\tilde{q}}$, as these searches do not rely on the jet emission from the gluino decay. In any case, figure 3 shows that the experimental strategies discussed in section 3.2 play complementary rolls in searching for long-lived gluinos, and by combining the results from these searches we can probe a wide range of parameter space in the compressed gluino scenario.

\section{Conclusion and discussion}

The compressed-gluino parameter region in the MSSM, where gluino and the LSP are highly degenerate in mass, can evade the over-abundance of dark matter and thus is still viable. Therefore, it is important to test this possibility experimentally. It is however difficult to probe this scenario using the conventional search strategy at the LHC based on the jets plus missing energy signatures, since jets emitted from the gluino decay tend to be very soft. In this paper, we have discussed the LHC search strategies which are sensitive to the compressed gluino scenario. They include the searches of DVs, disappearing tracks, and 
anomalous energy deposit in the Pixel detector, on top of the ordinary inclusive searches. Then we have found that these searches are indeed sensitive to long-lived gluinos.

In summary, we show the cover areas of these search strategies in the $\Delta m-c \tau_{\widetilde{g}}$ plane in figure 4, where we set the gluino mass to be $1.5 \mathrm{TeV}$ and consider the $13 \mathrm{TeV}$ LHC run with an integrated luminosity of $40 \mathrm{fb}^{-1}$. As seen in this figure, depending on the lifetime and the gluino-LSP mass difference, we can adopt different search strategies. When the gluino decay length is $\gtrsim 1 \mathrm{~m}$, the Pixel $d E / d x$ search offers the best sensitivity to gluinos. For $0.1 \mathrm{~m} \lesssim c \tau_{\tilde{g}} \lesssim 10 \mathrm{~m}$, the disappearing-track search is quite promising. The DV search can cover the range of $1 \mathrm{~mm} \lesssim c \tau_{\tilde{g}} \lesssim 1 \mathrm{~m}$, though its sensitivity strongly depends on the gluinoLSP mass difference. The $c \tau_{\tilde{g}}<1 \mathrm{~mm}$ region can be probed by ordinary prompt-decay gluino searches. As a consequence, these searches complement each other, which allows us to investigate a broad range of the compressed gluino region in the LHC experiments.

Notice that although we have focused on the cases with a high sfermion mass scale $(\widetilde{m} \gtrsim 10 \mathrm{TeV})$, the search strategies discussed in this paper, especially the disappearingtrack search and the $d E / d x$ search, can also be powerful for $\widetilde{m}<10 \mathrm{TeV}$ if the gluino-LSP mass difference is small enough to make gluino long-lived. Such a possibility may be interesting since it offers a refuge for (semi)natural SUSY models, which may be uncovered by the long-lived gluino searches.

In this paper, we discuss long-lived gluinos whose longevity comes from small mass difference between the gluino and the LSP. Actually, in not only the gluino case but more general "co-LSP" scenarios, we may have such a long-lived particle. For instance, in the very compressed stau-LSP and stop-LSP cases, metastable charged particles may appear and thus can be observed in the long-lived particle searches at the LHC. As it turns out, for instance, by using the setup of the disappearing track search discussed above, we can probe a right-handed stau (stop) with a mass of around 200 (950) GeV at the LHC Run2 for $c \tau=10 \mathrm{~cm}$. Detailed studies of such generalization are out of the scope of the present paper and will be discussed elsewhere.

\section{Acknowledgments}

We are grateful to M. Saito for helpful correspondence. The work of H.O. was supported by JSPS KAKENHI Grant Number 15K17653. The work of S.S. was supported by World Premier International Research Center Initiative (WPI), MEXT, Japan.

Open Access. This article is distributed under the terms of the Creative Commons Attribution License (CC-BY 4.0), which permits any use, distribution and reproduction in any medium, provided the original author(s) and source are credited.

\section{References}

[1] ATLAS collaboration, Further searches for squarks and gluinos in final states with jets and missing transverse momentum at $\sqrt{s}=13 \mathrm{TeV}$ with the ATLAS detector,

ATLAS-CONF-2016-078, CERN, Geneva Switzerland, (2016). 
[2] ATLAS collaboration, Search for pair production of gluinos decaying via top or bottom squarks in events with b-jets and large missing transverse momentum in pp collisions at $\sqrt{s}=13 \mathrm{TeV}$ with the ATLAS detector, ATLAS-CONF-2016-052, CERN, Geneva Switzerland, (2016).

[3] CMS collaboration, Search for new physics in the all-hadronic final state with the MT2 variable, CMS-PAS-SUS-16-015, CERN, Geneva Switzerland, (2016).

[4] ATLAS collaboration, Observation of a new particle in the search for the Standard Model Higgs boson with the ATLAS detector at the LHC, Phys. Lett. B 716 (2012) 1 [arXiv: 1207.7214] [INSPIRE].

[5] CMS collaboration, Observation of a new boson at a mass of $125 \mathrm{GeV}$ with the CMS experiment at the LHC, Phys. Lett. B 716 (2012) 30 [arXiv:1207.7235] [INSPIRE].

[6] ATLAS and CMS collaborations, Combined measurement of the Higgs boson mass in pp collisions at $\sqrt{s}=7$ and $8 T e V$ with the ATLAS and CMS experiments, Phys. Rev. Lett. 114 (2015) 191803 [arXiv:1503.07589] [INSPIRE].

[7] K. Inoue, A. Kakuto, H. Komatsu and S. Takeshita, Low-energy parameters and particle masses in a supersymmetric grand unified model, Prog. Theor. Phys. 67 (1982) 1889 [INSPIRE].

[8] R.A. Flores and M. Sher, Higgs masses in the standard, multi-Higgs and supersymmetric models, Annals Phys. 148 (1983) 95 [inSPIRE].

[9] Y. Okada, M. Yamaguchi and T. Yanagida, Upper bound of the lightest Higgs boson mass in the minimal supersymmetric Standard Model, Prog. Theor. Phys. 85 (1991) 1 [InSPIRE].

[10] Y. Okada, M. Yamaguchi and T. Yanagida, Renormalization group analysis on the Higgs mass in the softly broken supersymmetric Standard Model, Phys. Lett. B 262 (1991) 54 [INSPIRE].

[11] J.R. Ellis, G. Ridolfi and F. Zwirner, Radiative corrections to the masses of supersymmetric Higgs bosons, Phys. Lett. B 257 (1991) 83 [INSPIRE].

[12] H.E. Haber and R. Hempfling, Can the mass of the lightest Higgs boson of the minimal supersymmetric model be larger than $m_{Z}$ ?, Phys. Rev. Lett. 66 (1991) 1815 [INSPIRE].

[13] J.R. Ellis, G. Ridolfi and F. Zwirner, On radiative corrections to supersymmetric Higgs boson masses and their implications for LEP searches, Phys. Lett. B 262 (1991) 477 [INSPIRE].

[14] J.D. Wells, Implications of supersymmetry breaking with a little hierarchy between gauginos and scalars, hep-ph/0306127 [INSPIRE].

[15] J.D. Wells, PeV-scale supersymmetry, Phys. Rev. D 71 (2005) 015013 [hep-ph/0411041] [INSPIRE].

[16] N. Arkani-Hamed and S. Dimopoulos, Supersymmetric unification without low energy supersymmetry and signatures for fine-tuning at the LHC, JHEP 06 (2005) 073 [hep-th/0405159] [INSPIRE].

[17] G.F. Giudice and A. Romanino, Split supersymmetry, Nucl. Phys. B 699 (2004) 65 [Erratum ibid. B 706 (2005) 487] [hep-ph/0406088] [INSPIRE].

[18] N. Arkani-Hamed, S. Dimopoulos, G.F. Giudice and A. Romanino, Aspects of split supersymmetry, Nucl. Phys. B 709 (2005) 3 [hep-ph/0409232] [INSPIRE]. 
[19] L.J. Hall and Y. Nomura, Spread supersymmetry, JHEP 01 (2012) 082 [arXiv:1111.4519] [INSPIRE].

[20] L.J. Hall, Y. Nomura and S. Shirai, Spread supersymmetry with wino LSP: gluino and dark matter signals, JHEP 01 (2013) 036 [arXiv:1210.2395] [INSPIRE].

[21] M. Ibe, T. Moroi and T.T. Yanagida, Possible signals of wino LSP at the Large Hadron Collider, Phys. Lett. B 644 (2007) 355 [hep-ph/0610277] [INSPIRE].

[22] M. Ibe and T.T. Yanagida, The lightest Higgs boson mass in pure gravity mediation model, Phys. Lett. B 709 (2012) 374 [arXiv:1112.2462] [InSPIRE].

[23] M. Ibe, S. Matsumoto and T.T. Yanagida, Pure gravity mediation with $m_{3 / 2}=10-100 \mathrm{TeV}$, Phys. Rev. D 85 (2012) 095011 [arXiv:1202.2253] [INSPIRE].

[24] A. Arvanitaki, N. Craig, S. Dimopoulos and G. Villadoro, Mini-split, JHEP 02 (2013) 126 [arXiv: 1210.0555$]$ [INSPIRE].

[25] N. Arkani-Hamed, A. Gupta, D.E. Kaplan, N. Weiner and T. Zorawski, Simply unnatural supersymmetry, arXiv:1212.6971 [INSPIRE].

[26] J.L. Evans, M. Ibe, K.A. Olive and T.T. Yanagida, Universality in pure gravity mediation, Eur. Phys. J. C 73 (2013) 2468 [arXiv:1302.5346] [INSPIRE].

[27] J.L. Evans, K.A. Olive, M. Ibe and T.T. Yanagida, Non-universalities in pure gravity mediation, Eur. Phys. J. C 73 (2013) 2611 [arXiv:1305.7461] [InSPIRE].

[28] Y. Nomura and S. Shirai, Supersymmetry from typicality: TeV-scale gauginos and PeV-scale squarks and sleptons, Phys. Rev. Lett. 113 (2014) 111801 [arXiv:1407.3785] [INSPIRE].

[29] F. Gabbiani, E. Gabrielli, A. Masiero and L. Silvestrini, A complete analysis of FCNC and CP constraints in general SUSY extensions of the Standard Model, Nucl. Phys. B 477 (1996) 321 [hep-ph/9604387] [INSPIRE].

[30] T. Moroi and M. Nagai, Probing supersymmetric model with heavy sfermions using leptonic flavor and CP-violations, Phys. Lett. B 723 (2013) 107 [arXiv:1303.0668] [INSPIRE].

[31] D. McKeen, M. Pospelov and A. Ritz, Electric dipole moment signatures of PeV-scale superpartners, Phys. Rev. D 87 (2013) 113002 [arXiv: 1303.1172] [INSPIRE].

[32] W. Altmannshofer, R. Harnik and J. Zupan, Low energy probes of PeV scale sfermions, JHEP 11 (2013) 202 [arXiv:1308.3653] [InSPIRE].

[33] K. Fuyuto, J. Hisano, N. Nagata and K. Tsumura, QCD corrections to quark (chromo)electric dipole moments in high-scale supersymmetry, JHEP 12 (2013) 010 [arXiv: 1308.6493] [INSPIRE].

[34] J. Hisano, T. Kuwahara and N. Nagata, Grand unification in high-scale supersymmetry, Phys. Lett. B 723 (2013) 324 [arXiv:1304.0343] [INSPIRE].

[35] N. Sakai and T. Yanagida, Proton decay in a class of supersymmetric grand unified models, Nucl. Phys. B 197 (1982) 533 [inSPIRE].

[36] S. Weinberg, Supersymmetry at ordinary energies. 1. Masses and conservation laws, Phys. Rev. D 26 (1982) 287 [inSPIRE].

[37] T. Goto and T. Nihei, Effect of RRRR dimension five operator on the proton decay in the minimal SU(5) SUGRA GUT model, Phys. Rev. D 59 (1999) 115009 [hep-ph/9808255] [INSPIRE]. 
[38] H. Murayama and A. Pierce, Not even decoupling can save minimal supersymmetric SU(5), Phys. Rev. D 65 (2002) 055009 [hep-ph/0108104] [INSPIRE].

[39] M. Liu and P. Nath, Higgs boson mass, proton decay, naturalness and constraints of the LHC and Planck data, Phys. Rev. D 87 (2013) 095012 [arXiv:1303.7472] [InSPIRE].

[40] J. Hisano, D. Kobayashi, T. Kuwahara and N. Nagata, Decoupling can revive minimal supersymmetric $\mathrm{SU}(5)$, JHEP 07 (2013) 038 [arXiv:1304.3651] [INSPIRE].

[41] M. Dine, P. Draper and W. Shepherd, Proton decay at $M_{p l}$ and the scale of SUSY-breaking, JHEP 02 (2014) 027 [arXiv: 1308.0274] [INSPIRE].

[42] N. Nagata and S. Shirai, Sfermion flavor and proton decay in high-scale supersymmetry, JHEP 03 (2014) 049 [arXiv:1312.7854] [INSPIRE].

[43] L.J. Hall, Y. Nomura and S. Shirai, Grand unification, axion and inflation in intermediate scale supersymmetry, JHEP 06 (2014) 137 [arXiv:1403.8138] [INSPIRE].

[44] J.L. Evans, N. Nagata and K.A. Olive, SU(5) grand unification in pure gravity mediation, Phys. Rev. D 91 (2015) 055027 [arXiv: 1502.00034] [INSPIRE].

[45] B. Bajc, S. Lavignac and T. Mede, Resurrecting the minimal renormalizable supersymmetric SU(5) model, JHEP 01 (2016) 044 [arXiv: 1509.06680] [INSPIRE].

[46] J. Ellis, J.L. Evans, F. Luo, N. Nagata, K.A. Olive and P. Sandick, Beyond the CMSSM without an accelerator: proton decay and direct dark matter detection, Eur. Phys. J. C 76 (2016) 8 [arXiv: 1509.08838] [INSPIRE].

[47] J. Ellis, J.L. Evans, A. Mustafayev, N. Nagata and K.A. Olive, The super-GUT CMSSM revisited, Eur. Phys. J. C 76 (2016) 592 [arXiv: 1608.05370] [InSPIRE].

[48] N. Sakai, Naturalness in supersymmetric GUTs, Z. Phys. C 11 (1981) 153 [INSPIRE].

[49] S. Dimopoulos and H. Georgi, Softly broken supersymmetry and SU(5), Nucl. Phys. B 193 (1981) 150 [INSPIRE].

[50] H. Pagels and J.R. Primack, Supersymmetry, cosmology and new TeV physics, Phys. Rev. Lett. 48 (1982) 223 [INSPIRE].

[51] S. Weinberg, Cosmological constraints on the scale of supersymmetry breaking, Phys. Rev. Lett. 48 (1982) 1303 [INSPIRE].

[52] M. Yu. Khlopov and A.D. Linde, Is it easy to save the gravitino?, Phys. Lett. B 138 (1984) 265 [inSPIRE].

[53] M. Kawasaki, K. Kohri and T. Moroi, Big-bang nucleosynthesis and hadronic decay of long-lived massive particles, Phys. Rev. D 71 (2005) 083502 [astro-ph/0408426] [INSPIRE].

[54] M. Kawasaki, K. Kohri, T. Moroi and A. Yotsuyanagi, Big-bang nucleosynthesis and gravitino, Phys. Rev. D 78 (2008) 065011 [arXiv:0804.3745] [INSPIRE].

[55] G.D. Coughlan, W. Fischler, E.W. Kolb, S. Raby and G.G. Ross, Cosmological problems for the Polonyi potential, Phys. Lett. B 131 (1983) 59 [INSPIRE].

[56] Planck collaboration, P.A.R. Ade et al., Planck 2015 results. XIII. Cosmological parameters, Astron. Astrophys. 594 (2016) A13 [arXiv:1502.01589] [INSPIRE].

[57] J. Hisano, S. Matsumoto and M.M. Nojiri, Explosive dark matter annihilation, Phys. Rev. Lett. 92 (2004) 031303 [hep-ph/0307216] [INSPIRE]. 
[58] J. Hisano, S. Matsumoto, M.M. Nojiri and O. Saito, Non-perturbative effect on dark matter annihilation and gamma ray signature from galactic center, Phys. Rev. D 71 (2005) 063528 [hep-ph/0412403] [INSPIRE].

[59] J. Hisano, S. Matsumoto, M. Nagai, O. Saito and M. Senami, Non-perturbative effect on thermal relic abundance of dark matter, Phys. Lett. B 646 (2007) 34 [hep-ph/0610249] [INSPIRE].

[60] M. Cirelli, A. Strumia and M. Tamburini, Cosmology and astrophysics of minimal dark matter, Nucl. Phys. B 787 (2007) 152 [arXiv:0706.4071] [INSPIRE].

[61] T. Gherghetta, G.F. Giudice and J.D. Wells, Phenomenological consequences of supersymmetry with anomaly induced masses, Nucl. Phys. B 559 (1999) 27 [hep-ph/9904378] [INSPIRE].

[62] T. Moroi and L. Randall, Wino cold dark matter from anomaly mediated SUSY breaking, Nucl. Phys. B 570 (2000) 455 [hep-ph/9906527] [INSPIRE].

[63] N. Arkani-Hamed, A. Delgado and G.F. Giudice, The well-tempered neutralino, Nucl. Phys. B 741 (2006) 108 [hep-ph/0601041] [INSPIRE].

[64] S. Shirai, F. Takahashi and T.T. Yanagida, R-violating decay of wino dark matter and electron/positron excesses in the PAMELA/Fermi experiments, Phys. Lett. B 680 (2009) 485 [arXiv: 0905. 0388] [INSPIRE].

[65] M. Ibe, S. Matsumoto, S. Shirai and T.T. Yanagida, AMS-02 positrons from decaying wino in the pure gravity mediation model, JHEP 07 (2013) 063 [arXiv:1305.0084] [INSPIRE].

[66] M. Ibe, S. Matsumoto, S. Shirai and T.T. Yanagida, Mass of decaying wino from AMS-02 2014, Phys. Lett. B 741 (2015) 134 [arXiv:1409.6920] [InSPIRE].

[67] J. Hisano, K. Ishiwata and N. Nagata, QCD effects on direct detection of wino dark matter, JHEP 06 (2015) 097 [arXiv: 1504.00915] [INSPIRE].

[68] M. Ibe, S. Matsumoto and R. Sato, Mass splitting between charged and neutral winos at two-loop level, Phys. Lett. B 721 (2013) 252 [arXiv:1212.5989] [INSPIRE].

[69] T. Cohen, M. Lisanti, A. Pierce and T.R. Slatyer, Wino dark matter under siege, JCAP 10 (2013) 061 [arXiv: 1307.4082] [InSPIRE].

[70] J. Fan and M. Reece, In wino veritas? Indirect searches shed light on neutralino dark matter, JHEP 10 (2013) 124 [arXiv:1307.4400] [INSPIRE].

[71] A. Hryczuk, I. Cholis, R. Iengo, M. Tavakoli and P. Ullio, Indirect detection analysis: wino dark matter case study, JCAP 07 (2014) 031 [arXiv:1401.6212] [INSPIRE].

[72] B. Bhattacherjee, M. Ibe, K. Ichikawa, S. Matsumoto and K. Nishiyama, Wino dark matter and future dSph observations, JHEP 07 (2014) 080 [arXiv: 1405.4914] [INSPIRE].

[73] M. Cirelli, F. Sala and M. Taoso, Wino-like minimal dark matter and future colliders, JHEP 10 (2014) 033 [Erratum ibid. 01 (2015) 041] [arXiv:1407.7058] [INSPIRE].

[74] M. Baumgart, I.Z. Rothstein and V. Vaidya, Constraints on galactic wino densities from gamma ray lines, JHEP 04 (2015) 106 [arXiv: 1412.8698] [INSPIRE].

[75] K. Harigaya, K. Ichikawa, A. Kundu, S. Matsumoto and S. Shirai, Indirect probe of electroweak-interacting particles at future lepton colliders, JHEP 09 (2015) 105 [arXiv: 1504.03402] [INSPIRE]. 
[76] M. Ibe, S. Matsumoto, S. Shirai and T.T. Yanagida, Wino dark matter in light of the AMS-02 2015 data, Phys. Rev. D 91 (2015) 111701 [arXiv:1504.05554] [INSPIRE].

[77] K. Hamaguchi, T. Moroi and K. Nakayama, AMS-02 antiprotons from annihilating or decaying dark matter, Phys. Lett. B 747 (2015) 523 [arXiv: 1504.05937] [INSPIRE].

[78] V. Lefranc, E. Moulin, P. Panci, F. Sala and J. Silk, Dark matter in $\gamma$ lines: galactic center vs dwarf galaxies, JCAP 09 (2016) 043 [arXiv: 1608.00786] [INSPIRE].

[79] N. Nagata and S. Shirai, Higgsino dark matter in high-scale supersymmetry, JHEP 01 (2015) 029 [arXiv: 1410.4549] [InSPIRE].

[80] K. Cheung, C.-W. Chiang and J. Song, A minimal supersymmetric scenario with only $\mu$ at the weak scale, JHEP 04 (2006) 047 [hep-ph/0512192] [INSPIRE].

[81] K. Cheung, S.Y. Choi and J. Song, Impact on the light higgsino-LSP scenario from physics beyond the minimal supersymmetric Standard Model, Phys. Lett. B 677 (2009) 54 [arXiv:0903.3175] [INSPIRE].

[82] V.A. Beylin, V.I. Kuksa, G.M. Vereshkov and R.S. Pasechnik, Neutralino-nucleon interaction in the split higgsino scenario of the dark matter, Int. J. Mod. Phys. A 24 (2009) 6051 [arXiv: 0903.4201] [INSPIRE].

[83] K.S. Jeong, M. Shimosuka and M. Yamaguchi, Light higgsino in heavy gravitino scenario with successful electroweak symmetry breaking, JHEP 09 (2012) 050 [arXiv:1112.5293] [INSPIRE].

[84] P.J. Fox, G.D. Kribs and A. Martin, Split Dirac supersymmetry: an ultraviolet completion of higgsino dark matter, Phys. Rev. D 90 (2014) 075006 [arXiv:1405.3692] [InSPIRE].

[85] J.L. Evans, M. Ibe, K.A. Olive and T.T. Yanagida, Light higgsinos in pure gravity mediation, Phys. Rev. D 91 (2015) 055008 [arXiv: 1412.3403] [INSPIRE].

[86] E.J. Chun, S. Jung and J.-C. Park, Very degenerate higgsino dark matter, JHEP 01 (2017) 009 [arXiv: 1607.04288] [INSPIRE].

[87] A. Dedes, D. Karamitros and V.C. Spanos, Effective theory for electroweak doublet dark matter, Phys. Rev. D 94 (2016) 095008 [arXiv:1607.05040] [INSPIRE].

[88] K. Griest and D. Seckel, Three exceptions in the calculation of relic abundances, Phys. Rev. D 43 (1991) 3191 [INSPIRE].

[89] J.R. Ellis, T. Falk and K.A. Olive, Neutralino-stau coannihilation and the cosmological upper limit on the mass of the lightest supersymmetric particle, Phys. Lett. B 444 (1998) 367 [hep-ph/9810360] [INSPIRE].

[90] J.R. Ellis, T. Falk, K.A. Olive and M. Srednicki, Calculations of neutralino-stau coannihilation channels and the cosmologically relevant region of MSSM parameter space, Astropart. Phys. 13 (2000) 181 [Erratum ibid. 15 (2001) 413] [hep-ph/9905481] [INSPIRE].

[91] T. Nihei, L. Roszkowski and R. Ruiz de Austri, Exact cross-sections for the neutralino slepton coannihilation, JHEP 07 (2002) 024 [hep-ph/0206266] [INSPIRE].

[92] M. Citron, J. Ellis, F. Luo, J. Marrouche, K.A. Olive and K.J. de Vries, End of the CMSSM coannihilation strip is nigh, Phys. Rev. D 87 (2013) 036012 [arXiv:1212.2886] [INSPIRE].

[93] C. Boehm, A. Djouadi and M. Drees, Light scalar top quarks and supersymmetric dark matter, Phys. Rev. D 62 (2000) 035012 [hep-ph/9911496] [INSPIRE]. 
[94] J.R. Ellis, K.A. Olive and Y. Santoso, Calculations of neutralino stop coannihilation in the CMSSM, Astropart. Phys. 18 (2003) 395 [hep-ph/0112113] [INSPIRE].

[95] M.A. Ajaib, T. Li and Q. Shafi, Stop-neutralino coannihilation in the light of LHC, Phys. Rev. D 85 (2012) 055021 [arXiv: 1111.4467] [InSPIRE].

[96] J. Ellis, K.A. Olive and J. Zheng, The extent of the stop coannihilation strip, Eur. Phys. J. C 74 (2014) 2947 [arXiv: 1404.5571] [INSPIRE].

[97] A. De Simone, G.F. Giudice and A. Strumia, Benchmarks for dark matter searches at the LHC, JHEP 06 (2014) 081 [arXiv: 1402.6287] [INSPIRE].

[98] S.P. Liew and F. Luo, Effects of QCD bound states on dark matter relic abundance, JHEP 02 (2017) 091 [arXiv: 1611.08133] [INSPIRE].

[99] S. Profumo and C.E. Yaguna, Gluino coannihilations and heavy bino dark matter, Phys. Rev. D 69 (2004) 115009 [hep-ph/0402208] [INSPIRE].

[100] D. Feldman, Z. Liu and P. Nath, Gluino NLSP, dark matter via gluino coannihilation and LHC signatures, Phys. Rev. D 80 (2009) 015007 [arXiv:0905.1148] [INSPIRE].

[101] J. Ellis, F. Luo and K.A. Olive, Gluino coannihilation revisited, JHEP 09 (2015) 127 [arXiv: 1503.07142] [INSPIRE].

[102] J. Ellis, J.L. Evans, F. Luo and K.A. Olive, Scenarios for gluino coannihilation, JHEP 02 (2016) 071 [arXiv: 1510.03498] [inSPIRE].

[103] K. Harigaya, K. Kaneta and S. Matsumoto, Gaugino coannihilations, Phys. Rev. D 89 (2014) 115021 [arXiv:1403.0715] [INSPIRE].

[104] N. Nagata, H. Otono and S. Shirai, Probing bino-gluino coannihilation at the LHC, Phys. Lett. B 748 (2015) 24 [arXiv: 1504.00504] [INSPIRE].

[105] H. Baer, T. Krupovnickas, A. Mustafayev, E.-K. Park, S. Profumo and X. Tata, Exploring the BWCA (bino-wino co-annihilation) scenario for neutralino dark matter, JHEP 12 (2005) 011 [hep-ph/0511034] [INSPIRE].

[106] M. Ibe, A. Kamada and S. Matsumoto, Mixed (cold+warm) dark matter in the bino-wino coannihilation scenario, Phys. Rev. D 89 (2014) 123506 [arXiv:1311.2162] [INSPIRE].

[107] N. Nagata, H. Otono and S. Shirai, Probing bino-wino coannihilation at the LHC, JHEP 10 (2015) 086 [arXiv: 1506.08206] [inSPIRE].

[108] H.E. Haber and G.L. Kane, Gluino decays and experimental signatures, Nucl. Phys. B 232 (1984) 333 [INSPIRE].

[109] H. Baer, V.D. Barger, D. Karatas and X. Tata, Detecting gluinos at hadron supercolliders, Phys. Rev. D 36 (1987) 96 [inSPIRE].

[110] R. Barbieri, G. Gamberini, G.F. Giudice and G. Ridolfi, Constraining supergravity models from gluino production, Nucl. Phys. B 301 (1988) 15 [INSPIRE].

[111] H. Baer, X. Tata and J. Woodside, Phenomenology of gluino decays via loops and top quark Yukawa coupling, Phys. Rev. D 42 (1990) 1568 [INSPIRE].

[112] M. Toharia and J.D. Wells, Gluino decays with heavier scalar superpartners, JHEP 02 (2006) 015 [hep-ph/0503175] [INSPIRE].

[113] P. Gambino, G.F. Giudice and P. Slavich, Gluino decays in split supersymmetry, Nucl. Phys. B 726 (2005) 35 [hep-ph/0506214] [INSPIRE]. 
[114] R. Sato, S. Shirai and K. Tobioka, Gluino decay as a probe of high scale supersymmetry breaking, JHEP 11 (2012) 041 [arXiv:1207.3608] [INSPIRE].

[115] R. Sato, S. Shirai and K. Tobioka, Flavor of gluino decay in high-scale supersymmetry, JHEP 10 (2013) 157 [arXiv:1307.7144] [INSPIRE].

[116] W. Kilian, T. Plehn, P. Richardson and E. Schmidt, Split supersymmetry at colliders, Eur. Phys. J. C 39 (2005) 229 [hep-ph/0408088] [INSPIRE].

[117] J.L. Hewett, B. Lillie, M. Masip and T.G. Rizzo, Signatures of long-lived gluinos in split supersymmetry, JHEP 09 (2004) 070 [hep-ph/0408248] [INSPIRE].

[118] G.R. Farrar and P. Fayet, Phenomenology of the production, decay and detection of new hadronic states associated with supersymmetry, Phys. Lett. 76B (1978) 575 [INSPIRE].

[119] M. Fairbairn, A.C. Kraan, D.A. Milstead, T. Sjöstrand, P.Z. Skands and T. Sloan, Stable massive particles at colliders, Phys. Rept. 438 (2007) 1 [hep-ph/0611040] [INSPIRE].

[120] T. Sjöstrand, S. Mrenna and P.Z. Skands, PYTHIA 6.4 physics and manual, JHEP 05 (2006) 026 [hep-ph/0603175] [INSPIRE].

[121] A.C. Kraan, Interactions of heavy stable hadronizing particles, Eur. Phys. J. C 37 (2004) 91 [hep-ex/0404001] [INSPIRE].

[122] A. De Rujula, H. Georgi and S.L. Glashow, Hadron masses in a gauge theory, Phys. Rev. D 12 (1975) 147 [INSPIRE].

[123] M.S. Chanowitz and S.R. Sharpe, Spectrum of gluino bound states, Phys. Lett. 126B (1983) 225 [INSPIRE].

[124] UKQCD collaboration, M. Foster and C. Michael, Hadrons with a heavy color adjoint particle, Phys. Rev. D 59 (1999) 094509 [hep-lat/9811010] [InSPIRE].

[125] G.R. Farrar, Light gluinos, Phys. Rev. Lett. 53 (1984) 1029 [inSPIRE].

[126] F. Buccella, G.R. Farrar and A. Pugliese, $R$ baryon masses, Phys. Lett. B 153 (1985) 311 [INSPIRE].

[127] G.R. Farrar, R. Mackeprang, D. Milstead and J.P. Roberts, Limit on the mass of a long-lived or stable gluino, JHEP 02 (2011) 018 [arXiv:1011.2964] [INSPIRE].

[128] R. Mackeprang and A. Rizzi, Interactions of coloured heavy stable particles in matter, Eur. Phys. J. C 50 (2007) 353 [hep-ph/0612161] [INSPIRE].

[129] R. Mackeprang and D. Milstead, An updated description of heavy-hadron interactions in GEANT4, Eur. Phys. J. C 66 (2010) 493 [arXiv:0908.1868] [InSPIRE].

[130] ATLAS collaboration, The ATLAS experiment at the CERN Large Hadron Collider, 2008 JINST 3 S08003 [INSPIRE].

[131] ATLAS collaboration, ATLAS insertable B-layer technical design report, CERN-LHCC-2010-013, CERN, Geneva Switzerland, (2010).

[132] Z. Liu and B. Tweedie, The fate of long-lived superparticles with hadronic decays after LHC Run 1, JHEP 06 (2015) 042 [arXiv: 1503.05923] [INSPIRE].

[133] G. Chalons and D. Sengupta, Closing in on compressed gluino-neutralino spectra at the LHC, JHEP 12 (2015) 129 [arXiv: 1508.06735] [INSPIRE].

[134] A. Chakraborty, S. Chakraborty and T.S. Roy, Chasing new physics in stacks of soft tracks, Phys. Rev. D 94 (2016) 111703 [arXiv:1606.07826] [InSPIRE]. 
[135] A. Delgado, A. Martin and N. Raj, Extending the reach of compressed gluinos at the LHC, Phys. Rev. D 94 (2016) 115010 [arXiv: 1605.06479] [InSPIRE].

[136] ATLAS collaboration, Limits on metastable gluinos from ATLAS SUSY searches at $8 \mathrm{TeV}$, ATLAS-CONF-2014-037, CERN, Geneva Switzerland, (2014).

[137] ATLAS collaboration, Search for massive, long-lived particles using multitrack displaced vertices or displaced lepton pairs in pp collisions at $\sqrt{s}=8 \mathrm{TeV}$ with the ATLAS detector, Phys. Rev. D 92 (2015) 072004 [arXiv: 1504.05162] [InSPIRE].

[138] ATLAS collaboration, Search for charginos nearly mass degenerate with the lightest neutralino based on a disappearing-track signature in pp collisions at $\sqrt{s}=8 \mathrm{TeV}$ with the ATLAS detector, Phys. Rev. D 88 (2013) 112006 [arXiv:1310.3675] [INSPIRE].

[139] J.L. Feng, T. Moroi, L. Randall, M. Strassler and S.-F. Su, Discovering supersymmetry at the Tevatron in wino LSP scenarios, Phys. Rev. Lett. 83 (1999) 1731 [hep-ph/9904250] [INSPIRE].

[140] S. Asai, T. Moroi, K. Nishihara and T.T. Yanagida, Testing the anomaly mediation at the LHC, Phys. Lett. B 653 (2007) 81 [arXiv:0705.3086] [InSPIRE].

[141] S. Asai, T. Moroi and T.T. Yanagida, Test of anomaly mediation at the LHC, Phys. Lett. B 664 (2008) 185 [arXiv:0802.3725] [INSPIRE].

[142] S. Asai, Y. Azuma, O. Jinnouchi, T. Moroi, S. Shirai and T.T. Yanagida, Mass measurement of the decaying bino at the LHC, Phys. Lett. B 672 (2009) 339 [arXiv:0807.4987] [INSPIRE].

[143] ATLAS collaboration, Search for metastable heavy charged particles with large ionization energy loss in pp collisions at $\sqrt{s}=13 \mathrm{TeV}$ using the ATLAS experiment, Phys. Rev. D 93 (2016) 112015 [arXiv: 1604.04520] [inSPIRE].

[144] J. Alwall et al., The automated computation of tree-level and next-to-leading order differential cross sections and their matching to parton shower simulations, JHEP $\mathbf{0 7}$ (2014) 079 [arXiv: 1405.0301] [INSPIRE].

[145] T. Sjöstrand, S. Mrenna and P.Z. Skands, A brief introduction to PYTHIA 8.1, Comput. Phys. Commun. 178 (2008) 852 [arXiv:0710.3820] [INSPIRE].

[146] DELPHES 3 collaboration, J. de Favereau et al., DELPHES 3, a modular framework for fast simulation of a generic collider experiment, JHEP 02 (2014) 057 [arXiv:1307.6346] [INSPIRE].

[147] W. Beenakker, R. Hopker and M. Spira, PROSPINO: a program for the production of supersymmetric particles in next-to-leading order QCD, hep-ph/9611232 [INSPIRE].

[148] W. Beenakker, R. Hopker, M. Spira and P.M. Zerwas, Squark and gluino production at hadron colliders, Nucl. Phys. B 492 (1997) 51 [hep-ph/9610490] [INSPIRE].

[149] A. Kulesza and L. Motyka, Threshold resummation for squark-antisquark and gluino-pair production at the LHC, Phys. Rev. Lett. 102 (2009) 111802 [arXiv:0807.2405] [INSPIRE].

[150] A. Kulesza and L. Motyka, Soft gluon resummation for the production of gluino-gluino and squark-antisquark pairs at the LHC, Phys. Rev. D 80 (2009) 095004 [arXiv:0905.4749] [INSPIRE]. 
[151] W. Beenakker, S. Brensing, M. Krämer, A. Kulesza, E. Laenen and I. Niessen, Soft-gluon resummation for squark and gluino hadroproduction, JHEP 12 (2009) 041 [arXiv:0909.4418] [INSPIRE].

[152] W. Beenakker et al., Squark and gluino hadroproduction, Int. J. Mod. Phys. A 26 (2011) 2637 [arXiv:1105.1110] [INSPIRE].

[153] M. Saito, Search for direct electroweak gaugino production based on disappearing-track detection with four hits at LHC-ATLAS Run 2 (in Japanese), https://kds.kek.jp/indico/event/22451/session/15/contribution/185/material/slides/0.pdf.

[154] ATLAS collaboration, Search for charginos nearly mass degenerate with the lightest neutralino based on a disappearing-track signature in pp collisions at $\sqrt{s}=8 \mathrm{TeV}$ with the ATLAS detector, Phys. Rev. D 88 (2013) 112006 [arXiv:1310.3675] [INSPIRE].

[155] ATLAS collaboration, Search for metastable heavy charged particles with large ionization energy loss in pp collisions at $\sqrt{s}=13 \mathrm{TeV}$ using the ATLAS experiment, Phys. Rev. D 93 (2016) 112015 [arXiv: 1604.04520] [inSPIRE]. 